\title{
Bronchiolitis obliterans syndrome
}

\author{
Keith C. Meyer
}

Published online: 6 July 2012

(C) Springer Science+Business Media, LLC 2012

\begin{abstract}
Bronchiolitis obliterans syndrome (BOS) is thought to represent chronic allograft rejection. Primary graft dysfunction (PGD), acute cellular rejection (AR), lymphocytic bronchiolitis (LB), abnormal gastroesophageal reflux (GER) with microaspiration, and allograft infection have all been implicated as causes of BOS. Although BOS is generally considered to be caused by alloimmune responses to non-self tissue, more recent findings suggest that autoimmune responses to self-antigens and the triggering of innate immune responses to environment stimuli may play a significant role in the pathobiology of BOS. Effective treatment of BOS remains elusive, but azithromycin may stabilize and possibly improve FEV1 in patients who meet criteria for BOS, and gastric fundoplication may be beneficial if abnormal GER is detected. Augmented immunosuppression is generally ineffective, and other treatments such as total lymphoid irradiation (TLI) or extracorporeal photopheresis (ECP) may not have a significant impact on loss of function. Retransplantation can be considered in carefully selected patients.
\end{abstract}

Keywords Lung transplant · Bronchiolitis obliterans syndrome $\cdot$ Obliterative bronchiolitis $\cdot$ Chronic lung allograft dysfunction

\section{Introduction}

Bronchiolitis obliterans syndrome (BOS) represents the greatest threat to long-term survival following an initially

K. C. Meyer $(\square)$

Section of Allergy, Pulmonary and Critical Care Medicine,

Department of Medicine, University of Wisconsin

School of Medicine and Public Health,

Madison, WI, USA

e-mail: kcm@medicine.wisc.edu successful lung transplant (LTX). Loss of allograft function that meets criteria for the diagnosis of BOS (see Table 1 for current diagnostic criteria and staging) is attributed to the onset of a constrictive bronchiolitis that leads to airway fibrosis and disappearance of small airways due to luminal obliteration and cicatrix formation that has been termed obliterative bronchiolitis (OB) [1-4].

OB was first described in lung allograft tissues from heart-lung transplant recipients in 1984 [5] and was associated with delayed onset of declining allograft function and airflow obstruction following initial recovery from transplant and stabilization of graft function. OB was subsequently found to be a common histopathologic finding when delayed allograft dysfunction associated with a pattern of airflow obstruction on spirometric testing occurred in lung or heart-lung transplant recipients [6, 7]. However, definitive histopathologic changes are frequently absent when tissue sampling is relatively limited (despite the presence of $\mathrm{OB}$ ), due to the small size of the forceps biopsy specimens obtained via transbronchial lung biopsy (TBB), the patchy nature of the lesions, and bronchoscopistdependent sampling adequacy $[8,9]$. Because of the association of $\mathrm{OB}$ with an obstructive pattern of lung function decline and the difficulty of obtaining adequate lung tissue via $\mathrm{TBB}$ to make a confident diagnosis of $\mathrm{OB}$, FEV1 was chosen as a surrogate marker that could be used to indicate the likely presence of $\mathrm{OB}$ if other, potentially reversible causes of allograft functional decline are excluded as a cause of FEV1 decline [6].

Although a number of advances have been made in surgical techniques and early management that have improved early LTX outcomes, this has had little impact on the prevalence and outcome of BOS in LTX recipients. More than $50 \%$ of lung allograft recipients will develop clinically significant BOS [4], which is a common cause of graft loss and patient death. Strategies to prevent BOS as 
Table 1 Criteria for diagnosis and staging of BOS

\begin{tabular}{ll}
$\begin{array}{l}\text { BOS } \\
\text { grade }\end{array}$ & Spirometry (\% of baseline) \\
\hline 0 & $\mathrm{FEV}_{1}>90 \%$ of baseline and $\mathrm{FEF}_{25-75}>75 \%$ of baseline \\
$0-\mathrm{p}$ & $\mathrm{FEV}_{1} 81-90 \%$ of baseline and $/ \mathrm{orEF}_{25-75} \leq 75 \%$ of baseline \\
1 & $\mathrm{FEV}_{1} 66-80 \%$ of baseline \\
2 & $\mathrm{FEV}_{1} 51-65 \%$ of baseline \\
3 & $\mathrm{FEV}_{1} \leq 50 \%$ of baseline
\end{tabular}

Baseline defined as the average of the two best FEV1 determinations post-LTX obtained at least 3 weeks apart (J Heart Lung Transplant 2002;21:297-310)

well as strategies to detect it in early stages and effectively arrest it and prevent its progression and loss of allograft function are much needed to improve long-term survival following LTX.

\section{Terminology of lung allograft dysfunction}

The revised BOS classification system published in 2002 [10] states that lung function decline should be determined by comparing values obtained over time to an optimal, posttransplant baseline value (Table 1). The baseline value for FEV1 and FEF25-75 is the average of two highest values for each measurement obtained at least 3 weeks apart posttransplant (without the administration of a bronchodilator). By definition, 3 or more months are required to have elapsed from the date of the transplant for the diagnosis of BOS to be made to help distinguish BOS from acute and/or subacute complications of lung transplantation as well as taking into account the time needed to establish both a baseline FEV1 and the 3week interval needed to confirm a significant decline in FEV1 (via a second measurement) that meets the criteria for a diagnosis of BOS $[6,10]$. In practice, it may be difficult to establish a baseline with two reasonably concordant measurements 3 weeks apart due to various complications (e.g. acute rejection or infection) that may cause considerable undulation of FEV1 values over time, making it difficult to establish a stable baseline for some recipients. Due to concern that setting the cutoff value for FEV1 at $80 \%$ of the best post-transplant value may be insensitive to early decline in allograft function due to early $\mathrm{OB}$, the revised statement created stage BOS-0p ( $\geq 10 \%$ decline in FEV1 and/or $\geq 25 \%$ decline in FEF25-75) to signify "potential BOS" [10].

As our understanding of delayed lung allograft dysfunction has evolved over the past decade, it has become clear that many factors (e.g. acute rejection, infection, pleural disorders, anastomotic dysfunction, impaired allograft inflation, inflammatory disorders, recurrent parenchymal disease) may cause graft dysfunction beyond the acute post-transplant time period, and declining graft function that meets criteria for BOS may not necessarily be due to the development of clinically significant $\mathrm{OB}$. The terms chronic lung allograft dysfunction (CLAD) and chronic rejection have increasingly appeared in the literature and are frequently used synonymously or interchangeably with OB and BOS $[11 \bullet, 12 \bullet, 13 \bullet$. However, CLAD needs to be more rigorously defined and may be observed when entities other than $\mathrm{OB} / \mathrm{BOS}$ are present or when a combination of factors (which may include $\mathrm{OB}$ as one of the pathologic entities) negatively impact allograft function. Additionally, lung function decline consistent with a diagnosis of BOS can stabilize in some patients and not lead to sustained, progressive deterioration in allograft function and graft loss, as discussed below. Finally, the entity of restrictive allograft syndrome (RAS) has been recently described as a form of delayed allograft dysfunction characterized by parenchymal fibrosis that can be distinguished from typical BOS/OB $[11 \bullet, 13 \bullet, 14 \bullet \bullet]$; OB lesions may be present in lung specimens from recipients who develop this type of lesion, but restrictive physiology and radiographic parenchymal infiltrates are typically present (in contrast to $\mathrm{OB} / \mathrm{BOS}$, which is characterized by obstructive physiology and lacks significant parenchymal fibrosis).

\section{Phenotypes of BOS}

The identification of characteristics that identify subsets of BOS patients (differing disease mechanisms, risk factors, and responses to treatment interventions) may aid efforts to provide specific treatments and make key management decisions. Recipients with early onset BOS may represent a group that is prone to rapid progression and poor prognosis [15-18], although, some patients with rapidly declining lung function may stabilize despite an initial rapid onset and FEV1 decline [19]. Recent investigations have also described a phenotype of significant bronchoalveolar lavage (BAL) neutrophilia that is often associated with high-resolution computed tomographic (HRCT) changes of probable cellular bronchiolitis, and these individuals usually respond to azithromycin therapy [20•,21•]. Indeed, FEV1 may improve such that the recipient no longer meets spirometric criteria for BOS, and this entity has been labeled neutrophilic reversible allograft dysfunction (NRAD) $\left[1,20^{\bullet}\right]$. It has also been suggested that patients who meet BOS criteria but do not respond to azithromycin may represent a fibroproliferative OB phenotype [20•]. However, distinct phenotypes of BOS that are based upon specific risk factors or other parameters have yet to be definitively established.

\section{Pathogenesis and mechanisms of obliterative bronchiolitis}

Lung histopathology in patients with BOS shows striking similarities to the OB that can occur in allogeneic bone 
marrow or stem cell transplant recipients as well as constrictive bronchiolitis in patients with connective tissue diseases, and these airway changes are perceived as alloimmune or autoimmune disorders respectively. BOS is widely perceived as the physiological surrogate (FEV1 decline) of the effects of an alloimmune response to foreign tissue due to many observations that include its association with acute cellular rejection [22] and greater degrees of HLA mismatch [23]. Indeed, early acute allograft rejection and late/recurrent/refractory acute rejection and late lymphocytic bronchiolitis (LB) are prime risk factors (Table 2) for developing BOS [24-31]. Additionally, humoral rejection (e.g. de novo anti-HLA antibodies) has also been associated with the development of BOS [32, 33]. Nonetheless, although BOS is frequently equated with the term chronic rejection, various interventions including intensified immunosuppression may have little or no effect on the progressive loss of allograft function that is usually observed in transplant recipients who develop BOS.

More recent investigations have linked autoimmune sensitization to self-antigens (collagen $\mathrm{V}$ or K-alpha-1-tubulin) to BOS $[34,35 \cdot$, and alloimmune responses may stimulate or drive such autoimmune responses, which may involve Th17 cells and IL-17 [35•]. In addition to alloimmune and/ or autoimmune phenomena associated with BOS, many mechanisms that can be perceived as predominantly nonimmune have been implicated as playing a role in BOS pathogenesis. These include injury caused by primary graft dysfunction (PGD) [36, 37], gastroesophageal reflux (GER)

Table 2 Risk factors associated with the development of BOS

- Primary graft dysfunction

- Acute cellular rejection

- Lymphocytic bronchiolitis

- Humoral rejection (e.g. anti-HLA antibodies)

- Gastroesophageal reflux and (micro)aspiration

○ Acid reflux

- Non-acid reflux

- Infection

- Cytomegalovirus

- Community-acquired virus infection

- Bacterial colonization/infection (e.g. Pseudomonas)

○ Fungal colonization/infection (e.g. Aspergillus)

- Autoimmune sensitization to self-antigens

o Collagen V

$\circ \alpha$-k-tubulin

- Air pollution

- Inadequate recipient compliance with outpatient drug therapies

- Other potential risks

- Accelerated allograft aging due to cellular senescence

- Ischemic airway injury due to disrupted bronchial microcirculation with microaspiration [38, 39], airway ischemia caused by disruption of the bronchial circulation [40 $]$, and infections caused by viruses [41, 42], bacteria [43, 44], or fungi [45]. These "non-immune" factors may promote tissue inflammation that in turn initiates and may intensify an alloimmune rejection response or autoimmune reaction to lung airway self-antigens. Finally, it has been suggested that environmental exposures may lead to airway injury and obliteration, and higher ambient levels of pollutants have been linked to BOS in lung transplant recipients [46]. Indeed, these "nonimmune" stimuli may induce BOS by triggering innate immune responses [3], which may then also interact with cellular and humoral arms of adaptive immunity that determine alloimmune and autoimmune responses against nonself (e.g. MHC mismatched allograft antigens) as well as allograft self-antigens (e.g. collagen V).

$\mathrm{OB} / \mathrm{BOS}$ pathogenesis is thought to involve a primary stimulus that activates resident airway dendritic cells and/or airway macrophages, which leads to up-regulation of chemokines and cytokines of epithelial and endothelial origin. This local inflammation attracts and activates additional inflammatory cells, especially lymphocytes and neutrophils, and also provokes the production of cytokines by structural airway cells such as myofibroblasts and smooth muscle cells. Interleukin-17-producing cells (Th17 cells) have been linked to the constrictive bronchiolitis of BOS and may be the prime stimulus for upregulation of IL- 8 , which is a potent chemoattractant of neutrophils [47, 48]. As airway inflammation progresses, epithelial and interstitial damage is likely induced by reactive oxygen intermediate production and matrix metalloproteinase (MMP) production [49, 50]. Epithelial-mesenchymal transition can occur in response to epithelial damage and lead to the fibroproliferative changes that cause airway scarring and obliteration [51, 52•], and allograft fibroproliferative responses may be driven by resident mesenchymal cells [53]. Additionally, accelerated senescence of epithelial or other allograft cell types may play a role in predisposing the allograft to developing chronic airway dysfunction and BOS [54].

Because established OB displays variable evidence of inflammation, alloimmune reactions, autoimmunity, and fibroproliferation with airway obliteration that leads to allograft airway remodeling and loss of function, OB may well represent a final common end-point for allograft bronchiolar injury precipitated and/or driven by a variety of insults and mechanisms. Indeed, innate immune responses to a variety of environmental insults (LPS from airway bacteria, refluxed non-acid gastric contents that contain LPS due to gram-negative bacterial overgrowth, viral infection, fungal colonization, or air pollution) via toll-like receptor (TLR)mediated pathways may play an important role in the induction and progression of BOS, and some LTX recipients may have genetic polymorphisms (e.g. TLR4, CD14) that make 
them more susceptible to triggering of innate immune responses [3]. As mentioned above, although BOS is frequently equated with the term chronic rejection, intensified immunosuppression may have little or no effect on the progressive FEV1 decline that is commonly observed unless a response occurs to treatments such as azithromycin.

\section{Bronchoalveolar lavage neutrophils and BOS}

Persistent increases in BAL neutrophils have been observed in patients with allograft complications such as infection, acute rejection, $\mathrm{LB}$, and established BOS $[55,56]$, and $\mathrm{BAL}$ neutrophilia has been linked to BOS [57-59] and identified as a predictor of increased overall mortality risk [60]. As mentioned above, Vos et al. [20•] have shown that recipients who meet criteria for BOS but have significantly increased numbers of neutrophils in BAL (generally greater than $15 \%$ on differential cell count) are likely to respond to azithromycin therapy and may regain function such that BOS criteria are no longer met. HRCT scans in these patients tend to show changes consistent with cellular bronchiolitis that clear with effective azithromycin therapy.

\section{Diagnosis of BOS}

Once patients stabilize following transplantation, their clinic visits gradually decrease in frequency if they have recovered well and are clinically stable. However, patients need to be educated to recognize and promptly report symptoms that may indicate that respiratory or other complications are developing. Follow-up clinic visits typically include spirometry and routine chest radiographs, and a 6-MWT may also be performed. Some studies suggest that a decline in FEV1 that meets the FEV1 criterion for BOS Stage 0p (a 10-19\% decline in FEV1 from baseline) correlates with an increased risk of evolving OB/BOS [61-63]. The lower sensitivity and specificity of the $\mathrm{FEF}_{25-75}$ criterion for BOS Stage 0p ( $\geq 25 \%$ decline), however, appears to make a decline in this measurement less reliable for predicting the early onset of evolving $\mathrm{OB} / \mathrm{BOS}$. In addition to routine imaging and pulmonary function testing, surveillance bronchoscopies are performed by a majority of transplant centers in the US [64], especially during the first year after transplant, and BAL and transbronchial biopsies performed when surveillance bronchoscopy is performed can frequently detect occult infection or rejection [65]. There is general consensus that prompt and effective treatment of these entities (even when patients are clinically stable) may decrease the risk of developing BOS.

Many centers have patients perform once or twice daily home spirometry to track FEV1 following successful lung transplant and subsequent stabilization of graft function, and a decline in FEV1 needs to be reported to the transplant center so that a prompt evaluation can be undertaken. Concern for possible BOS should trigger an evaluation to determine the cause of lung function decline. In addition to a clinical evaluation with history and physical examination, a routine chest radiograph and spirometry are typically obtained. Additionally, the 6-MWT test can be useful to detect a significant decline in walk distance and/or significant oxyhemoglobin desaturation that was not present on previous clinic evaluations. Bronchoscopy is typically performed with BAL and transbronchial biopsy to rule out acute rejection or infection as a cause, and biopsy specimens may occasionally be obtained that are diagnostic of OB. HRCT imaging may be useful to detect abnormalities for which the routine chest radiograph is insensitive, and HRCT imaging with expiratory imaging may reveal changes of cellular bronchiolitis, bronchiectasis and/or air trapping consistent with bronchiolitis [66-68]. A thorough evaluation can detect rejection, infection, or other causes of delayed lung function decline that meets the definition of BOS. It should be kept in mind that more than one pathologic process may simultaneously affect the lung allograft, and detection of initial stages of allograft dysfunction due to evolving BOS may be somewhat difficult in patients who receive single LTX for pulmonary fibrosis. Although many potentially useful biomarkers have been examined and reported, a biomarker that possesses good sensitivity and specificity to predict the presence of BOS versus other causes of declining lung function has yet to be found.

\section{Management of BOS}

Considerations for the management of BOS are given in Table 3. Because BOS has been perceived as representing a form of allograft rejection and the administration of high-dose corticosteroids is a standard treatment measure for newly detected AR that can usually reverse an acute decline in lung function and stabilize the patient, intensified immunosuppression has been viewed as a logical strategy for treating BOS. However, there are no data to support intensified doses of corticosteroids as an effective therapy for BOS, and one study reported a lack of response to repetitive administration of pulsed, high-dose methylprednisolone [69]. Additionally, chronic or pulse high-dose corticosteroid administration is associated with numerous, significant adverse side effects and is not likely to stabilize or improve the course of BOS. Other treatments such as the administration of cytolytic agents, the addition or cytotoxic drugs such as cyclophosphamide or methotrexate, the use of aerosolized cyclosporine A, or the addition of an mTOR inhibitor (e.g. sirolimus or everolimus) have not been shown to have significant beneficial 
Table 3 Management of BOS

\footnotetext{
- Exclude other, potentially treatable/reversible causes of impaired graft function

- Administration of chronic azithromycin

- Consider adjusting maintenance immunosuppression

- Switch to tacrolimus if on CSA-based regimen

$\circ$ Avoid sustained, high-dose corticosteroids

- Evaluate for abnormal GER (acid and non-acid)

- Consider fundoplication if significant GER is present

- Consider IVIG, plasma exchange, and/or rituximab if de novo anti-HLA antigen detected

- Progressive BOS refractory to other interventions

$\circ$ Total lymphoid irradiation

- Extracorporeal photopheresis

$\circ$ Retransplantation
}

CSA cyclosporine A; GER gastroesophageal reflux; IVIG intravenous immunoglobulin

effect [70]. However, a number of case series suggest that if a patient is not receiving tacrolimus as their calcineurin inhibitor, switching from CSA to tacrolimus may stabilize declining lung function $[69,71,72]$. If a significant level of one or more de novo anti-HLA antibodies is detected, intravenous immunoglobulin, plasmaphoresis, and or anti-B-cell therapy (e.g. rituximab) may prove beneficial [70].

\section{Azithromycin}

Macrolides and neo-macrolides such as azithromycin (an azalide) possess anti-inflammatory effects and can inhibit IL-8 production and neutrophil recruitment, suppress bronchial inflammation, and prevent airway damage for a number of respiratory disorders [73]. Many centers have reported that a substantial number of patients who develop BOS respond to azithromycin and may have their lung function stabilized or significantly improve such that some patients may no longer meet FEV1 criteria for BOS as they respond to the drug [74•, 75]. Notably, azithromycin has been reported to diminish the risk of graft loss and recipient death when given to patients with established BOS $\left[20^{\bullet}, 76^{\bullet}\right]$. The recently published, randomized prospective clinical trial conducted by Vos et al. [77••] suggested that prophylactic administration of azithromycin initiated shortly after transplantation can significantly decrease the risk of developing BOS, although a significant impact on survival was not shown over the relatively brief, 2year evaluation period.

Gastroesophageal reflux and (micro)aspiration

Abnormal GER is highly prevalent in patients with advanced lung disease and in LTX recipients [78, 79], and the prevalence may increase post-transplant [80, 81]. Notably, abnormal acid GER has been strongly linked to risk for BOS. However, pharmacologic therapy with proton-pump inhibitors (PPI), although increased $\mathrm{pH}$ of gastric secretions induced by PPI therapy may relieve symptoms, may have little effect on GER [82]. It should be noted that the majority of studies in patients with advanced lung disease and LTX recipients only used $\mathrm{pH}$ monitoring and did not utilize impedance to detect weakly acid or non-acid reflux, which could contain bile acids, for one. Additionally, only recent studies have measured pepsin and bile acids in BAL fluid obtained at bronchoscopy, which can identify those patients in whom microaspiration of refluxed foregut secretions is actually taking place. Indeed, PPI therapy may have little effect on nonacid reflux, which typically contains bile acids that can be very injurious to the lung $[82,83]$. Additionally, increased gastric $\mathrm{pH}$ induced by PPI therapy may allow some degree of bacterial overgrowth, and LPS from gram-negative bacteria, if present, may induce mucosal inflammation if aspirated [84]. Interestingly, non-acid reflux with bile acids has been linked to BOS and to chronic Pseudomonas colonization/infection [85], suggesting that bile acids aspirated into the lower respiratory tract may be particularly injurious to respiratory mucosae and induce airway injury and dysfunction consistent with BOS as well as chronic bacterial infection, which is also a risk factor associated with BOS. The situation can be complicated by the presence of significant esophageal motility disorders that are typical of patients with pulmonary fibrosis due to connective tissue disorders such as scleroderma [86], and aspiration of ingested foods may also lead to airway injury and BOS in LTX recipients. In addition to foregut dysmotility, swallowing disorders are not uncommon post-transplant [87], although the relationship of oropharyngeal dysphagia to BOS risk is unclear. Lastly, GER may promote autoimmune sensitization to self-antigens and increase risk of BOS via sensitization to collagen $\mathrm{V}[88 \bullet$.

Because pharmacologic suppression of gastric acid secretion may not significantly suppress abnormal GER (especially weakly acid or non-acid reflux) and microaspiration, gastric fundoplication has been investigated as means of preventing LTX complications and as a treatment for BOS when reflux appears to be present. A number of investigations have shown that fundoplication can be safely performed $[89,90,91 \cdot]$, and case series suggest that it may prevent the appearance of BOS or prevent its progression if abnormal GER is diagnosed in patients who have developed BOS [92]. Additionally, it may lead to improved lung function such that patients can revert to BOS Stage 0 [93], but prospective, randomized trials have not been reported. Interestingly, azithromycin therapy has been shown to decrease GER and microaspiration in lung transplant recipients [94], but it appears to have reduced efficacy when patients with BOS have evidence of bile acid aspiration [95•], suggesting that fundoplication is likely the best approach to 
managing GER in these patients, particularly if bile acids are present in BAL fluid.

\section{Other therapies}

Augmentation of immunosuppression via total lymphoid irradiation (TLI) or extracorporeal photopheresis (ECPP) have been evaluated by a number of centers. These therapies attempt to reduce the numbers of sensitized lymphocytes that may be driving immunologically-mediated chronic rejection and causing BOS. Fisher et al. [96] reported significant reduction in the rate of FEV1 decline for 27 recipients who completed at least $80 \%$ of the radiation fractions (pre-TLI $123 \mathrm{ml} /$ month decline vs. $25 \mathrm{ml} /$ month decline post-TLI); major adverse effects included bone marrow suppression and infection. Limited data suggest that ECPP may reverse, stabilize or decrease the rate of FEV1 decline in some patients with BOS [97], but the mechanism of action is unclear. Postulated mechanisms to explain how ECPP modifies host responses include induction of a clone-specific anti-lymphocyte immune response, altered cytokine production, and/or induction of regulatory T-cells. ECPP is generally well-tolerated but expensive. Potential risks include infectious complications and/or bone marrow suppression. If bronchiectasis and chronic infection becomes established in recipients with evolving BOS, infections (e.g. P. aeruginosa) need to be treated and suppressed.

\section{Retransplantation}

Retransplantation may be the only treatment option for progressive BOS that is refractory to other forms of treatment. A number of single-center retrospective analyses have been reported, and actuarial survival has gradually improved [98, 99]. Outcomes after retransplantation appear to be better when performed for BOS versus outcomes for PGD or other complications, and outcomes following retransplantation for carefully selected patients (ambulatory patients evaluated via the same selection process used for first-time transplantation) with BOS are expected to approach those of first-time lung transplants if performed by experienced centers. Various ethical questions regarding access to the scarce resource of donor lungs (taking utility and equity into consideration) must be considered carefully when retransplantation is considered for refractory BOS or other allograft complications.

\section{Prevention of BOS}

It is generally agreed that LTX recipients should be maintained on triple immunotherapy in the form of a CNI (tacrolimus or CSA), an antimetabolite (mycophenolic acid or azathioprine) and corticosteroids (gradually tapered to low/minimal dose). The data for this is mostly derived from other forms of solid organ transplantation. However, this approach is generally accepted for LTX recipients as it is well recognized that the lungs are the most immunogenic of the solid organs with a greater propensity for both acute and chronic allograft rejection. The best forms and combinations of maintenance therapy are unknown, but effective immunosuppression that can prevent AR, LB, or humoral rejection are essential. Many centers also administer an anti-lymphocyte antibody in the immediate postoperative period, although the impact on BOS incidence is marginal per the International Society for Lung Transplantation (ISHLT) Registry. An alternative to the antimetabolites are the mTOR inhibitors (sirolimus or everolimus), and a mTOR inhibitor can be used in their place or added to allow a decrease in calcineurin inhibitor dose. The calcineurin and mTOR inhibitors are metabolized by CYP3A4 (cytochrome P-450), and care must be taken to avoid drug-drug interactions if multiple CYP3A4-metabolized agents are used simultaneously. If preLTX anti-HLA antibodies are detected, cross-matching should be done to avoid implanting a mismatched allograft. Lastly, bronchial arteries are typically not re-anastomosed at the time of LTX, and it remains unknown whether re-establishing the bronchial circulation at the time of transplant can prevent subsequent BOS.

Rapid diagnosis and prompt/effective treatment of AR, LB, antibody-mediated rejection, or infection may not only prevent acute complications but also prevent the delayed appearance of BOS. Prevention of infection via prophylaxis (CMV, Pneumocystis jiroveci, Aspergillus) may also help prevent later BOS. Additionally, peri-operative targeted antibacterial therapy for bacterial isolates from respiratory secretions of patients with $\mathrm{CF}$ as well as non-CF recipients with bronchiectasis followed by sustained suppression (e.g. inhaled antipseudomonal antibiotics) may help prevent early untoward events but also diminish risk of delayed allograft dysfunction due to BOS. The prospective, randomized, placebo-controlled trial of azithromycin begun shortly after transplant suggest that such may prevent BOS/NRAD. However, whether LTX recipients should be placed on early azithromycin for BOS prophylaxis following successful LTX requires further investigation and discussion. If patients develop BOS and are not receiving azithromycin, available evidence strongly supports the initiation of chronic azithromycin therapy. Additionally, abnormal GER should be considered and ruled out via appropriate investigations. If GER is present, anti-reflux surgery (e.g. laparoscopic fundoplication) should be considered; if such surgery is indicated, it should be performed by a surgeon who is skilled in anti-reflux surgery.

\section{New directions and research needs}

Multicenter trials are needed to better establish optimal regimens for the induction and maintenance of immunosuppression 
that can adequately prevent both acute and chronic allograft dysfunction yet not lead to excessive risk for infection or other untoward consequences. Testing that can identify patients who are more tolerant to their grafts and, therefore, require less intense immunosuppression is also needed. Induction of tolerance to self-antigens (e.g. collagen $\mathrm{V}$ ) or strategies to augment regulatory $\mathrm{T}$ or $\mathrm{B}$ cells to promote and maintain tolerance may diminish risk for BOS. Guidelines for optimal testing for abnormal GER and the selection of patients (and procedure) for antireflux surgery to prevent or treat BOS need to be established, and, optimal approaches to allograft surveillance (e.g. the role of bronchoscopy with transbronchial biopsies in clinically stable LTX recipients, screening for de novo anti-HLA antibodies and the presence of humoral rejection) need to be determined. Finally, the effects of allograft cell senescence (accelerated aging) on allograft function and BOS risk need to be examined, and a better understanding of IL-17 and neutrophil responses as well as mechanisms by which EMT leads to airway fibrosis is needed. Placebo-controlled, preferably multicenter clinical trials that evaluate newer therapies to prevent or treat BOS (e.g. administration of pirfenidone, vitamin D supplemental, administration of montelukast or a combination of montelukast/azithromycin/inhaled corticosteroids as is used for bone marrow transplant recipients with $\mathrm{OB}$, etc.) are needed.

Acknowledgments Support was provided in part by the George and Julie Mosher Pulmonary Research Fund.

Disclosure No potential conflicts of interest relevant to this article were reported.

\section{References}

Papers of particular interest, published recently, have been highlighted as:

- of importance

•- of major importance

1. Verleden GM, Vos R, de Vleeschauwer SI, Willems-Widyastuti A, Verleden SE, Dupont LJ, Van Raemdonck DE, Vanaudenaerde BM. Obliterative bronchiolitis following lung transplantation: from old to new concepts? Transpl Int. 2009;22:771-9.

2. Kinnier CV, Martinu T, Palmer SM. Bronchiolitis obliterans syndrome: clinical risk factors and pathophysiology. In Lung Transplantation. Edited by Vigneswaran WT \& Garrity ED Jr. London: Informa; 2010:328-335.

3. Todd JL, Palmer SM. Bronchiolitis obliterans syndrome: the final frontier for lung transplantation. Chest. 2011;140:502-8.

4. Christie JD, Edwards LB, Aurora P, et al. The Registry of the International Society for Heart and Lung Transplantation: Twentysixth Official Adult Lung and Heart-Lung Transplantation Report2009. J Heart Lung Transplant. 2009;28:1031-49.

5. Burke CM, Theodore J, Dawkins KD, et al. Post-transplant obliterative bronchiolitis and other late lung sequelae in human heartlung transplantation. Chest. 1984;86:824-9.
6. Cooper JD, Billingham M, Egan T, et al. A working formulation for the standardization of nomenclature and for clinical staging of chronic dysfunction in lung allografts. International Society for Heart and Lung Transplantation. J Heart Lung Transplant. 1993;12:713-6.

7. Sharples LD, McNeil K, Stewart S, Wallwork J. Risk factors for bronchiolitis obliterans: a systematic review of recent publications. J Heart Lung Transplant. 2002;21:271-81.

8. Kramer MR, Stoehr C, Whang JL, et al. The diagnosis of obliterative bronchiolitis after heart-lung and lung transplantation: low yield of transbronchial lung biopsy. J Heart Lung Transplant. 1993; 12:675-81.

9. Chamberlain D, Maurer J, Chaparro C, Idolor L. Evaluation of transbronchial lung biopsy specimens in the diagnosis of bronchiolitis obliterans after lung transplantation. J Heart Lung Transplant. 1994;13:963-71.

10. Estenne M, Maurer JR, Boehler A, et al. Bronchiolitis obliterans syndrome 2001: an update of the diagnostic criteria. J Heart Lung Transplant. 2002;21:297-310.

11. - Verleden GM, Vos R, Verleden SE. Survival determinants in lung transplant patients with chronic allograft dysfunction. Transplantation. 2011;92:703-8. This study suggests that different phenotypes of CLAD (chronic lung allograft dysfunction) can be identified and that prognosis and survival differs among CLAD phenotypes.

12. - Verleden SE, Vos R, Mertens V. Heterogeneity of chronic lung allograft dysfunction: insights from protein expression in bronchoalveolar lavage. J Heart Lung Transplant. 2011;30:667-73. This study found heterogeneity in BAL inflammatory mediator levels that may be useful in distinguishing CLAD phenotypes.

13. - Woodrow JP, Shlobin OA, Barnett SD, Burton N, Nathan SD. Comparison of bronchiolitis obliterans syndrome to other forms of chronic lung allograft dysfunction after lung transplantation. J Heart Lung Transplant. 2010;29:1159-64. This study identified a subset of patients with BOS plus radiographic infiltrates as well as some BOS patients with a restrictive spirometric pattern.

14. • Sato M, Waddell TK, Wagnetz U. Restrictive allograft syndrome (RAS): a novel form of chronic lung allograft dysfunction. J Heart Lung Transplant. 2011;30:735-42. This study identified a subset of patients with CLAD (irreversible decline of $\geq 20 \%$ in FEV1 from baseline) plus restriction (defined as a $\geq 10 \%$ decline in total lung capacity from baseline) and the term "restrictive allograft syndrome" (RAS) was coined to differential this group from patients with traditional BOS (CLAD without RAS).

15. Lama VN, Murray S, Lonigro RJ, et al. Course of FEV(1) after onset of bronchiolitis obliterans syndrome in lung transplant recipients. Am J Respir Crit Care Med. 2007;175:1192-8.

16. Jackson CH, Sharples LD, McNeil K, Stewart S, Wallwork J. Acute and chronic onset of bronchiolitis obliterans syndrome (BOS): are they different entities? J Heart Lung Transplant. 2002;21:658-66.

17. Brugière $\mathrm{O}$, Pessione $\mathrm{F}$, Thabut $\mathrm{G}$, Mal H, Jebrak $\mathrm{G}$, Lesèche $\mathrm{G}$, Fournier M. Bronchiolitis obliterans syndrome after single-lung transplantation: impact of time to onset on functional pattern and survival. Chest. 2002;121(6):1883-9.

18. Burton CM, Carlsen J, Mortensen J, Andersen CB, Milman N, Iversen M. Long-term survival after lung transplantation depends on development and severity of bronchiolitis obliterans syndrome. J Heart Lung Transplant. 2007;26:681-6.

19. Nathan SD, Ross DJ, Belman MJ, et al. Bronchiolitis obliterans in single-lung transplant recipients. Chest. 1995;107:967-72.

20. • Vos R, Vanaudenaerde BM, Ottevaere A. Long-term azithromycin therapy for bronchiolitis obliterans syndrome: divide and conquer? J Heart Lung Transplant. 2010;29:1358-68. This study found that chronic azithromycin can be an effective therapy (improved lung function and survival) for patients who develop BOS, especially when BAL neutrophilia is present. 
21. Gottlieb J, Szangolies J, Koehnlein T. Long-term azithromycin for bronchiolitis obliterans syndrome after lung transplantation. Transplantation. 2008;85:36-41. This study reported that chronic azithromycin can improve lung function for a significant number of patients, and BAL neutrophilia was predictive of a beneficial response.

22. Burton CM, Iversen M, Carlsen J, et al. Acute cellular rejection is a risk factor for bronchiolitis obliterans syndrome independent of post-transplant baseline FEV1. J Heart Lung Transplant. 2009;28:888-93.

23. Schulman LL, Weinberg AD, McGregor C, et al. Mismatches at the HLA-DR and HLA-B loci are risk factors for acute rejection after lung transplantation. Am J Respir Crit Care Med. 1998; 157:1833-7.

24. Girgis RE, Tu I, Berry GJ, et al. Risk factors for the development of obliterative bronchiolitis after lung transplantation. J Heart Lung Transplant. 1996;15:1200-8.

25. Kroshus TJ, Kshettry VR, Savik K, et al. Risk faactors for the development of bronchiolitis obliterans syndrome after lung transplantation. J Thorac Cardiovasc Surg. 1997;114:195-202.

26. Husain AN, Siddiqui MT, Holmes EW, et al. Analysis of risk factors for the development of bronchiolitis obliterans syndrome. Am J Respir Crit Care Med. 1999;159(3):829-33.

27. Heng D, Sharples LD, McNeil K, et al. Bronchiolitis obliterans syndrome: incidence, natural history, prognosis, and risk factors. J Heart Lung Transplant. 1998;17:1255-63.

28. Burton CM, Iversen M, Scheike T, Carlsen J, Andersen CB. Is lymphocytic bronchiolitis a marker of acute rejection? An analysis of 2,697 transbronchial biopsies after lung transplantation. J Heart Lung Transplant. 2008;27:1128-34.

29. Ross DJ, Marchevsky A, Kramer M, Kass RM. "Refractoriness" of airflow obstruction associated with isolated lymphocytic bronchiolitis/bronchitis in pulmonary allografts. J Heart Lung Transplant. 1997; 16:832-8.

30. Glanville AR, Aboyoun CL, Havryk A, et al. Severity of lymphocytic bronchiolitis predicts long-term outcome after lung transplantation. Am J Respir Crit Care Med. 2008;177:1033-40.

31. Vos R, Vanaudenaerde BM, Verleden SE, et al. Bronchoalveolar lavage neutrophilia in acute lung allograft rejection and lymphocytic bronchiolitis. J Heart Lung Transplant. 2010;29:1259-69.

32. Palmer SM, Davis RD, Hadjiliadis D, et al. Development of an antibody specific to major histocompatibility antigens detectable by flow cytometry after lung transplant is associated with bronchiolitis obliterans syndrome. Transplantation. 2002;74:799-804.

33. Girnita AL, Duquesnoy R, Yousem SA, et al. HLA-specific antibodies are risk factors for lymphocytic bronchiolitis and chronic lung allograft dysfunction. Am J Transplant. 2005;5:131-8.

34. Burlingham WJ, Love RB, Jankowska-Gan E, et al. IL-17dependent cellular immunity to collagen type $\mathrm{V}$ predisposes to obliterative bronchiolitis in human lung transplants. J Clin Invest. 2007; 117:3498-506.

35. - Saini D, Weber J, Ramachandran S. Alloimmunity-induced autoimmunity as a potential mechanism in the pathogenesis of chronic rejection of human lung allografts. J Heart Lung Transplant. 2011;30:624-31. This study linked the development of donorspecific HLA antibodies to the induction of autoimmune sensitization to self-antigens $(K-\alpha 1$ tubulin \& collagen $V)$ with activation of the IL-17 pathway.

36. Daud SA, Yusen RD, Meyers BF, et al. Impact of immediate primary lung allograft dysfunction on bronchiolitis obliterans syndrome. Am J Respir Crit Care Med. 2007;175:507-13.

37. Huang HJ, Yusen RD, Meyers BF, et al. Late primary graft dysfunction after lung transplantation and bronchiolitis obliterans syndrome. Am J Transplant. 2008;8(11):2454-62.

38. Hadjiliadis D, Duane Davis R, Steele MP. Gastroesophageal reflux disease in lung transplant recipients. Clin Transplant. 2003;17:363-8.
39. D’Ovidio F, Mura M, Tsang M, et al. Bile acid aspiration and the development of bronchiolitis obliterans after lung transplantation. J Thorac Cardiovasc Surg. 2005;129:1144-52.

40. - Dhillon GS, Zamora MR, Roos JE. Lung transplant airway hypoxia: a diathesis to fibrosis? Am J Respir Crit Care Med. 2010;182:230-6. This study found that proximal lung allograft airways in single lung transplant show evidence of airway hypoxia distal to the allograft bronchial anastomosis.

41. Keenan RJ, Lega ME, Dummer JS, et al. Cytomegalovirus serologic status and postoperative infection correlated with risk of developing chronic rejection after pulmonary transplantation. Transplantation. 1991;51:433-8.

42. Khalifah AP, Hachem RR, Chakinala MM, et al. Respiratory viral infections are a distinct risk for bronchiolitis obliterans syndrome and death. Am J Respir Crit Care Med. 2004;170:181-7.

43. Vos R, Vanaudernaerde BM, De Vleeschauwer SI, et al. De novo or persistent pseudomonal airway colonization after lung transplantation: importance for bronchiolitis obliterans syndrome? Transplantation. 2008;86:624-5.

44. Gottlieb J, Mattner F, Weissbrodt H. Impact of graft colonization with gram-negative bacteria after lung transplantation on the development of bronchiolitis obliterans syndrome in recipients with cystic fibrosis. Respir Med. 2009;103:743-9.

45. Weigt SS, Elashoff RM, Huang C, et al. Aspergillus colonization of the lung allograft is a risk factor for bronchiolitis obliterans syndrome. Am J Transplant. 2009;9:1903-11.

46. Nawrot TS, Vos R, Jacobs L, et al. The impact of traffic air pollution on bronchiolitis obliterans syndrome and mortality after lung transplantation. Thorax. 2011;66:748-54.

47. Vanaudenaerde BM, De Vleeschauwer SI, Vos R, et al. The role of the IL23/IL17 axis in bronchiolitis obliterans syndrome after lung transplantation. Am J Transplant. 2008;8:1911-20.

48. DiGiovine B, Lynch 3rd JP, Martinez FJ, et al. Bronchoalveolar lavage neutrophilia is associated with obliterative bronchioliltis after lung transplantation: role of IL-8. J Immunol. 1996;157:4194-202.

49. Banerjee B, Ling KM, Sutanto EN, et al. The airway epithelium is a direct source of matrix degrading enzymes in bronchiolitis obliterans syndrome. J Heart Lung Transplant. 2011;30:1175-85.

50. Hirsch J, Elssner A, Mazur G, et al. Bronchiolitis obliterans syndrome after (heart-)lung transplantation. Impaired antiprotease defense and increased oxidant activity. Am J Respir Crit Care Med. 1999;160:1640-6.

51. Borthwick LA, Parker SM, Brougham KA, et al. Epithelial to mesenchymal transition (EMT) and airway remodelling after human lung transplantation. Thorax. 2009;64:770-7.

52. - Badri L, Murray S, Liu LX. Mesenchymal stromal cells in bronchoalveolar lavage as predictors of bronchiolitis obliterans syndrome. Am J Respir Crit Care Med. 2011;183:1062-70. This study found that BAL mesenchymal colony-forming units were significantly increased when lung histopathology showed either bronchiolitis obliterans or organizing pneumonia.

53. Walker N, Badri L, Wettlaufer S, et al. Resident tissue-specific mesenchymal progenitor cells contribute to fibrogenesis in human lung allografts. Am J Pathol. 2011;178:2461-9.

54. Parker SM, Goriwiec MR, Borthwick LA, et al. Airway epithelial cell senescence in the lung allograft. Am J Transplant. 2008;8:1544-9.

55. Meyer KC, Nunley DR, Dauber JH, et al. Neutrophils, unopposed neutrophil elastase, and alpha1-antiprotease defenses following human lung transplantation. Am J Respir Crit Care Med. 2001;164:97-102.

56. Reynaud-Gaubert M, Marin V, Thirion X, et al. Upregulation of chemokines in bronchoalveolar lavage fluid as a predictive marker of post-transplant airway obliteration. J Heart Lung Transplant. 2002;21:721-30. 
57. Zheng L, Whitford HM, Orsida B, et al. The dynamics and associations of airway neutrophilia post lung transplantation. Am J Transplant. 2006;6:599-608.

58. Schloma J, Slebos DJ, Boezen HM, et al. Eosinophilic granulocytes and interleukin-6 level in bronchoalveolar lavage fluid are associated with the development of obliterative bronchiolitis after lung transplantation. Am J Respir Crit Care Med. 2000;162:2221-5.

59. Neurohr C, Huppmann P, Samweber B, et al. Prognostic value of bronchoalveolar lavage neutrophilia in stable lung transplant recipients. J Heart Lung Transplant. 2009;28:468-74.

60. Henke JA, Golden JA, Henke JA, Golden JA, Yelin EH. Persistent increase of BAL neutrophils as a predictor of mortality following lung transplant. Chest. 1999;115:403-9.

61. Nathan SD, Barnett SD, Wohlrab J, Burton N. Bronchiolitis obliterans syndrome: utility of the new guidelines in single lung transplant recipients. J Heart Lung Transplant. 2003;22:427-32.

62. Hachem RR, Chakinala MM, Yusen RD, Lynch JP, Aloush AA, Patterson GA, Trulock EP. The predictive value of bronchiolitis obliterans syndrome stage 0-p. Am J Respir Crit Care Med. 2004;169:468-72.

63. Lama VN, Murray S, Mumford JA, Flaherty KR, Chang A, Toews GB, Peters-Golden M, Martinez FJ. Prognostic value of bronchiolitis obliterans syndrome stage 0 -p in single-lung transplant recipients. Am J Respir Crit Care Med. 2005;172:379-83.

64. Levine SM. Transplant/Immunology Network of the American College of Chest Physicians. A survey of clinical practice of lung transplantation in North America. Chest. 2004;125:1224-38.

65. Glanville AR. The role of bronchoscopic surveillance monitoring in the care of lung transplant recipients. Semin Respir Crit Care Med. 2006;27:480-91.

66. Collins J. Imaging of the chest after lung transplantation. J Thorac Imaging. 2002;17:102-12.

67. Bankier AA, Van Muylem A, Knoop C, Estenne M, Gevenois PA. Bronchiolitis obliterans syndrome in heart-lung transplant recipients: diagnosis with expiratory CT. Radiology. 2001;218:533-9.

68. Leung AN, Fisher K, Valentine V, Girgis RE, Berry GJ, Robbins $\mathrm{RC}$, Theodore J. Bronchiolitis obliterans after lung transplantation: detection using expiratory HRCT. Chest. 1998;113:365-70.

69. Ross DJ, Lewis MI, Kramer M, Vo A, Kass RM. FK 506 'rescue' immunosuppression for obliterative bronchiolitis after lung transplantation. Chest. 1997;112:1175-9.

70. Shah PD, Orens JB. Bronchiolitis obliterans: diagnosis and management. In Lung Transplantation. Edited by Vigneswaran WT \& Garrity ED Jr. London: Informa; 2010:336-346.

71. Fieguth HG, Krueger S, Wiedenmann DE, Otterbach I, Wagner TO. Tacrolimus for treatment of bronchiolitis obliterans syndrome after unilateral and bilateral lung transplantation. Transplant Proc. 2002;34:1884.

72. Cairn J, Yek T, Banner NR, Khaghani A, Hodson ME, Yacoub M. Time-related changes in pulmonary function after conversion to tacrolimus in bronchiolitis obliterans syndrome. J Heart Lung Transplant. 2003;22:50-7.

73. Kanoh S, Rubin BK. Mechanisms of action and clinical application of macrolides as immunomodulatory medications. Clin Microbiol Rev. 2010;23:590-615.

74. • Vos R,Vanaudenaerde BM,Verleden SE, et al. Antiinflammatory and immunomodulatory properties of azithromycin involved in treatment and prevention of chronic lung allograft rejection. Transplantation 2012 Mar 28. [Epub ahead of print]. This manuscript provides an excellent review of the antiinflammatory and immunomodulatory properties of azithromycin that may explain its benefit for patients who develop BOS.

75. Verleden GM, Vanaudenaerde BM, Dupont LJ, Van Raemdonck DE. Azithromycin reduces airway neutrophilia and interleukin- 8 in patients with bronchiolitis obliterans syndrome. Am J Respir Crit Care Med. 2006;174:566-70.
76. • Jain R, Hachem RR, Morrell MR, Trulock EP, Chakinala MM, Yusen RD, Huang HJ, Mohanakumar T, Patterson GA, Walter MJ. Azithromycin is associated with increased survival in lung transplant recipients with bronchiolitis obliterans syndrome. J Heart Lung Transplant. 2010;29:531-7. This study found that if azithromycin was given to patients with Stage 1 BOS, the risk of death was significantly reduced.

77. • Vos R, Vanaudenaerde BM, Verleden SE, De Vleeschauwer SI, Willems-Widyastuti A, Van Raemdonck DE, Schoonis A, Nawrot TS, Dupont LJ, Verleden GM. A randomized placebo-ccontrolled trial of azithromycin to prevent bronchiolitis obliterans syndrome after lung transplantation. Eur Respir J. 2011;37:164-72. This prospective, randomized, placebo-controlled clinical trial showed that patients who received prophylactic azithromycin following transplant were less likely to develop BOS.

78. D'Ovidio F, Singer LG, Hadjiliadis D, Pierre A, Waddell TK, de Perrot M, et al. Prevalence of gastroesophageal reflux in end-stage lung disease candidates for lung transplant. Ann Thorac Surg. 2005;80:1254-60.

79. Murthy SC, Nowicki ER, Mason DP. Pretransplant gastroesophageal reflux compromises early outcomes after lung transplantation. J Thorac Cardiovasc Surg. 2011;142(1):47-52.e3.

80. Young LR, Hadjiliadis D, Davis RD, Palmer SM. Lung transplantation exacerbates gastroesophageal reflux disease. Chest. 2003;124:1689-93.

81. D’Ovidio F, Mura M, Tsang M, Waddell TK, Hutcheon MA, Singer LG, Hadjiliadis D, Chaparro C, Gutierrez C, Pierre A, Darling G, Liu M, Keshavjee S. Bile acid aspiration and the development of bronchiolitis obliterans after lung transplantation. J ThoracCardiovasc Surg. 2005;129:1144-52.

82. Blondeau K, Mertens V, Vanaudenaerde BA, Verleden GM, Van Raemdonck DE, Sifrim D, et al. Gastro-oesophageal reflux and gastric aspiration in lung transplant patients with or without chronic rejection. Eur Respir J. 2008;31:707-13.

83. D'Ovidio F, Mura M, Ridsdale R, Takahashi H, Waddell TK, Hutcheon $\mathrm{M}$, et al. The effect of reflux and bile acid aspiration on the lung allograft and its surfactant and innate immunity molecules SP-A and SP-D. Am J Transplant. 2006;6:1930-8.

84. Mertens V, Blondeau K, Vanaudenaerde B, Vos R, Farre R, Pauwels A, et al. Gastric juice from patients "on" acid suppressive therapy can still provoke a significant inflammatory reaction by human bronchial epithelial cells. J Clin Gastroenterol. 2010;44: e230-235.

85. Vos R, Blondeau K, Vanaudenaerde BM, Mertens V, Van Raemdonck $\mathrm{DE}$, Sifrim D, et al. Airway colonization and gastric aspiration after lung transplantation: do birds of a feather flock together? J Heart Lung Transplant. 2008;27:843-9.

86. Patti MG, Gasper WJ, Fisichella PM, Nipomnick I, Palazzo F. Gastroesophageal reflux disease and connective tissue disorders: pathophysiology and implications for treatment. J Gastrointest Surg. 2008;12:1900-6.

87. Atkins BZ, Petersen RP, Daneshmand MA, Turek JW, Lin SS, Davis Jr RD. Impact of oropharyngeal dysphagia on longterm outcomes of lung transplantation. Ann Thorac Surg. 2010;90:1622-8.

88. • Bobadilla JL, Jankowska-Gan E, Xu Q, Haynes LD, Munoz del Rio A, Meyer K. Reflux-induced collagen type $\mathrm{v}$ sensitization: potential mediator of bronchiolitis obliterans syndrome. Chest. 2010;138:363-70. This study found an association between posttransplant gastroesophageal reflux and sensitization to collagen $V$.

89. Linden PA, Gilbert RJ, Yeap BY, Boyle K, Deykin A, Jaklitsch MT, et al. Laparoscopic fundoplication in patients with end-stage lung disease awaiting transplantation. J Thorac Cardiovasc Surg. 2006;131:438-46.

90. Gasper WJ, Sweet MP, Hoopes C, Leard LE, Kleinhenz ME, Hays $\mathrm{SR}$, et al. Antireflux surgery for patients with end-stage lung 
disease before and after lung transplantation. Surg Endosc. 2008;22:495-500.

91. • Hoppo T, Jarido V, Pennathur A, Morrell M, Crespo M, Shigemura $\mathrm{N}$, et al. Antireflux surgery preserves lung function in patients with gastroesophageal reflux disease and end-stage lung disease before and after lung transplantation. Arch Surg 2011;146:1041-1047. This study found that gastroesophageal reflux including laryngopharyngeal reflux events were common in both pre- and post-transplant patients and frequently asymptomatic, and anti-reflux surgery (ARS) stabilized the majority of patients (both pre- and posttransplant) with declining FEV1 prior to ARS.

92. Cantu 3rd E, Appel 3rd JZ, Hartwig MG, Woreta H, Green C, Messier R. J. Maxwell Chamberlain Memorial Paper. Early fundoplication prevents chronic allograft dysfunction in patients with gastroesophageal reflux disease. Ann Thorac Surg. 2004;78:114251

93. Davis Jr RD, Lau CL, Eubanks S, Messier RH, Hadjiliadis D, Steele MP, et al. Improved lung allograft function after fundoplication in patients with gastroesophageal reflux disease undergoing lung transplantation. J Thorac Cardiovasc Surg. 2003;125:533-42.

94. Mertens V, Blondeau K, Pauwels A, Farre R, Vanaudenaerde B, Vos $\mathrm{R}$, et al. Azithromycin reduces gastroesophageal reflux and aspiration in lung transplant recipients. Dig Dis Sci. 2009;54:9729.

95. - Mertens V, Blondeau K, Van Oudenhove L, Vanaudenaerde B, Vos R, Farre R. Bile acids aspiration reduces survival in lung transplant recipients with BOS despite azithromycin. Am J Transplant. 2011;11:329-35. This study found that lung transplant recipients with evidence of bile acid aspiration on BAL analysis had a significantly worse outcome despite therapy with azithromycin.

96. Fisher AJ, Rutherford RM, Bozzino J, Parry G, Dark JH, Corris PA. The safety and efficacy of total lymphoid irradiation in progressive bronchiolitis obliterans syndrome after lung transplantation. Am J Transplant. 2005;5:537-43.

97. Marques MB, Schwartz J. Update on extracorporeal photopheresis in heart and lung transplantation. J Clin Apher. 2011;26:146-51.

98. Novick RJ, Stitt LW, Al-Kattan K, Klepetko W, Schäfers HJ, Duchatelle JP, Khaghani A, Hardesty RL, Patterson GA, Yacoub MH. Pulmonary retransplantation: predictors of graft function and survival in 230 patients. Pulmonary Retransplant Registry. Ann Thorac Surg. 1998;65:227-34.

99. Keshavjee S. Lung Retransplantation Comes of Age. J Thorac Cardiovasc Surg. 2006;132:226-8. 